\title{
How I do it - MRT der Hand
}

Rainer Schmitt

\section{HINWEIS}

Die Beiträge in der Rubrik „How I do it“ sind als Anregung und Orientierungshilfe zu verstehen. Es bleibt jeder/jedem Kollegin/Kollegen überlassen, von diesem Schema abzuweichen, sofern man sich mit der Indikationsfrage befasst hat und sachliche Gründe für ein alternatives Vorgehen sprechen.

\section{Einleitung}

Die Magnetresonanztomografie (MRT) der Hand gilt als herausfordernd, weil sie einerseits eine Vielzahl anatomischer Strukturen auf kleinstem Raum abbilden muss und sich andererseits viele Krankheitsentitäten an der Hand manifestieren können.

Anatomisch wird die Hand von 37 Knochen gebildet, die über unterschiedlich geformte Gelenke untereinander in Verbindung stehen. Artikuläre Besonderheiten betreffen die komplexen Gelenkflächen der Handwurzel, die zusammen mit einem subtilen Bandapparat multiaxiale Freiheitsgrade bei gleichzeitig hoher Stabilität gewährleisten, sowie die Greiffunktion der Hand mit gerichteter Konvergenz der Finger auf das Skaphoid und der Opposition des Daumens. Dabei fungiert die Handwurzel als zwischengeschaltetes Glied („intercalated segment“) zwischen Unterarm und Mittelhand, da die extrinsischen Muskeln des Unterarms erst an den Mittelhand- bzw. Fingerknochen inserieren.

Ein weiteres Charakteristikum an der Hand ist die hohe Dichte synovialer Hüllstrukturen an den Gelenken sowie der Flexoren- und Extensorensehnen. Synovialitiden können nicht nur durch lokale Friktionen und Verletzungen entstehen, sondern auch Manifestation systemischer Erkrankungen sein.

\section{Untersuchungstechnische Voraussetzungen}

Für das diagnostische Ziel, auch kleinste Strukturen im „Mikrokosmos“ der Hand nachzuweisen und zu charakterisieren, stehen aktuell untersuchungstechnisch folgende Hardware- und Software-Optionen zur Verfügung:
- In der muskuloskelettalen Diagnostik werden aufgrund des höheren Signal-Rausch-Verhältnisses 3-Tesla-Scanner gegenüber 1,5-Tesla-Geräten bevorzugt [1].

- Dezidierte Mehrkanal-Gelenkspulen in Phased-ArrayTechnik steigern die Ortsauflösung im Untersuchungsvolumen und verkürzen zudem durch die parallele Datenakquisition die Messzeit [2]. Aktuell werden 16-Kanal-Phased-Array-Spulen verwendet, zukünftig werden Spulen mit 32 und mehr Kanälen zum Einsatz kommen.

- Durch die hohe Dichte synovialen Gewebes kann der Umgebungskontrast an der Hand durch die intravenöse oder intraartikuläre Kontrastmittelapplikation gesteigert werden [3]. Auf weitere Indikationen zur Gadoliniumgabe wird später eingegangen.

- Die sorgfältige Wahl der Schichtebenen und Sequenztypen auf der Grundlage der klinischen Fragestellung und der anatomischen Zielstruktur ist für die korrekte Diagnose entscheidend.

\section{MRT-Untersuchungstechnik}

Die wichtigsten Kriterien zur Untersuchungsplanung sind in $>$ Abb. 1 zusammengefasst, die Empfehlungen zu den Sequenzprotokollen in > Tab. 1 bis Tab. 3 .

\section{Lagerung}

Wegen der besseren Homogenität der Flussdichte $B_{0}$ im Zentrum des Scanners ist die „in-center“-Positionierung der Hand vorteilhaft. Die häufig jungen Handpatienten tolerieren die „Superman“-Position in Bauchlage über ca. 20 Minuten meist problemlos. Es handelt sich um eine Lagerung in Pronationsstellung, bei der 2 diagnostische Fallstricke bekannt sein müssen: Zum einen kommt es zu einer relativen Plusvarianz der Ulna (Vorsicht bei der Längenbestimmung), zum anderen zur relativen Extension des Lunatums (Capitatum verbleibt kolinear zum Radius) [4]. Bei schlanken oder immobilen Patienten und großer Tunnelöffnung kann die MRT-Untersuchung „off-center“ in Rückenlage mit der Hand seitlich der Hüfte („Habachtstellung“) durchgeführt werden, was der Neutralstellung entspricht. 


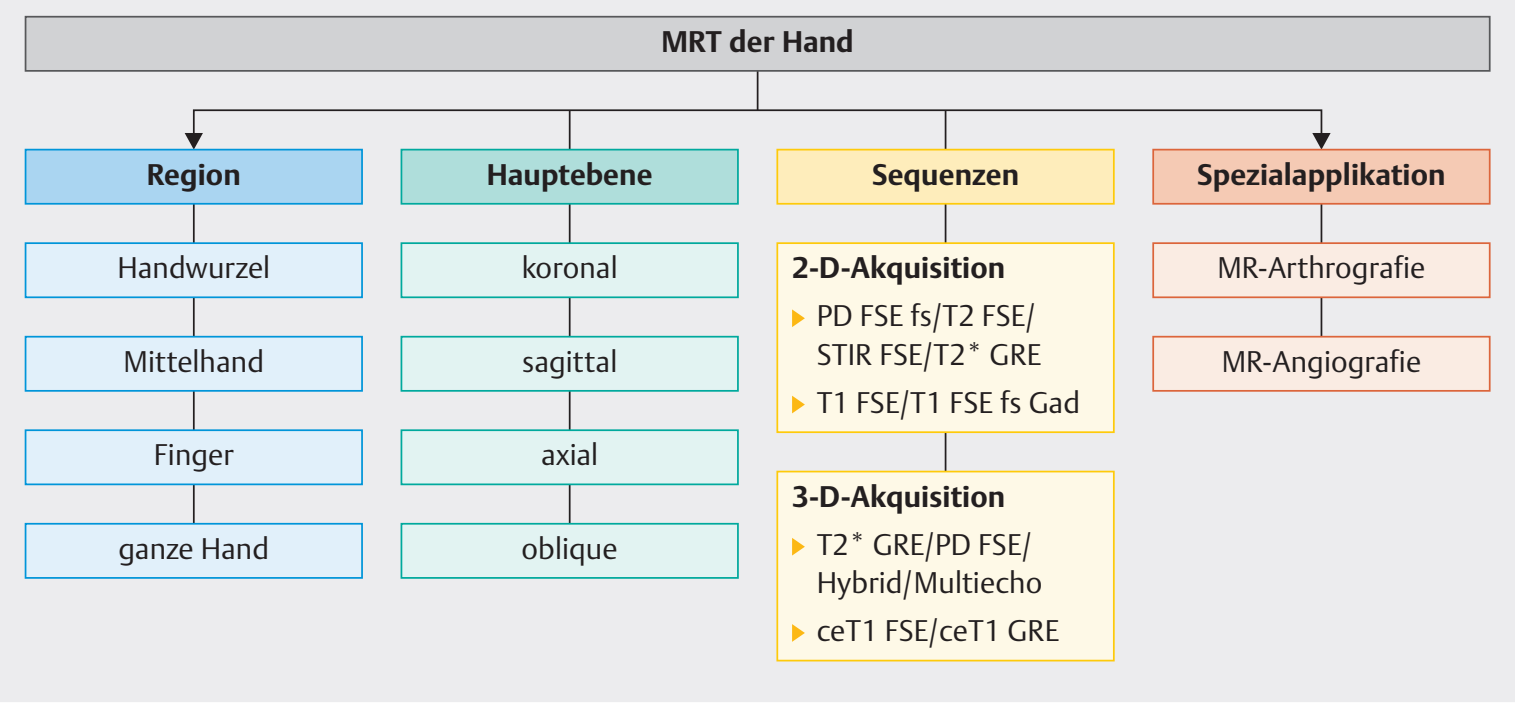

- Abb. 1 Vorgehen bei der Planung einer MRT-Untersuchung der Hand.

- Tab. 1 Empfehlungen zur MRT der Handwurzel. 2-D-Schichtdicke von 2,0 koronal (lückenlos) sowie 2,5 oder 3,0 mm axial und sagittal (am Lunatum 2,0 mm). 3-D-Partitionsdicken von jeweils 0,5 mm für die DESS- und VIBE-Sequenz.

\begin{tabular}{|c|c|c|c|c|c|c|c|}
\hline Protokoll & Indikationen an der Handwurzel & $\begin{array}{l}\text { PD FSE } \\
\text { fs }\end{array}$ & $\begin{array}{l}\text { T1 FSE } \\
\text { nativ }\end{array}$ & $\begin{array}{l}\text { PD FSE fs } \\
\text { oder T2* } \\
\text { GRE }\end{array}$ & $\begin{array}{l}\text { T1 FSE fs } \\
\text { nach i.v. } \\
\text { Gadolinium }\end{array}$ & $\begin{array}{l}\text { T1 FSE fs } \\
\text { nach i. v. } \\
\text { Gadolinium }\end{array}$ & optional \\
\hline 1 & $\begin{array}{l}\text { Übersicht, Ligament-, TFCC- } \\
\text { Veränderungen, Arthritis }\end{array}$ & koronal & koronal & axial & koronal & sagittal & $\begin{array}{l}\text { 3-D- } \\
\text { DESS }\end{array}$ \\
\hline 2 & $\begin{array}{l}\text { Ganglien, Tumoren, } \\
\text { Neuropathien }{ }^{2}\end{array}$ & koronal $^{3}$ & axial & axial $^{3}$ & axial & sagittal & - \\
\hline 3 & Tendopathien ${ }^{4}$ & koronal & axial & sagittal & axial & koronal & $\begin{array}{l}\text { 3-D- } \\
\text { VIBE }\end{array}$ \\
\hline 4 & $\begin{array}{l}\text { Skaphoidfraktur, Skaphoid- } \\
\text { pseudarthrose }^{6}\end{array}$ & koronal & $\begin{array}{l}\text { schräg- } \\
\text { sagittal }\end{array}$ & $\begin{array}{l}\text { schräg- } \\
\text { sagittal }\end{array}$ & $\begin{array}{l}\text { schräg- } \\
\text { sagittal }\end{array}$ & $\begin{array}{l}\text { schräg- } \\
\text { koronal }\end{array}$ & - \\
\hline 5 & $\begin{array}{l}\text { Lunatumnekrose, Impaktation, } \\
\text { intraossäres Ganglion }\end{array}$ & koronal & koronal & sagittal ${ }^{7}$ & koronal & sagittal & - \\
\hline 6 & MR-Arthrografie & koronal & koronal & axial & - & - & $\begin{array}{l}\text { 3-D- } \\
\text { VIBE }\end{array}$ \\
\hline \multicolumn{8}{|c|}{${ }^{1}$ empfohlen bei TFCC-Läsionen: Akquisition koronal, MPR radiär (Drehzentrum im Processus styloideus ulnae) } \\
\hline \multicolumn{8}{|c|}{${ }^{2}$ gilt auch für Ganglien, Tumoren und Neuropathien der Mittelhand } \\
\hline \multicolumn{8}{|c|}{${ }^{3}$ vorzugsweise T2-FSE-Sequenz ohne/mit Fettsättigung verwenden } \\
\hline \multicolumn{8}{|c|}{${ }^{4}$ gilt auch für Tendopathien der Mittelhand } \\
\hline \multicolumn{8}{|c|}{${ }^{5}$ empfohlen bei schräg verlaufenden Sehnen (z. B. EPL, EDM): Akquisition koronal, MPR einfach oder doppelt anguliert } \\
\hline \multicolumn{8}{|c|}{${ }^{6}$ parallel zur Längsachse des Skaphoids ausrichten } \\
\hline \multicolumn{8}{|c|}{${ }^{7}$ Schichtdicke $2 \mathrm{~mm}$, auf das Lunatum begrenzt } \\
\hline \multicolumn{8}{|c|}{${ }^{8}$ empfohlen bei jeder MR-Arthrografie: Akquisition koronal, MPR axial, koronal, sagittal und schräg anguliert } \\
\hline \multicolumn{8}{|c|}{$\begin{array}{l}\mathrm{PD}=\text { Protonendichte, } \mathrm{fs}=\text { „fat saturated“, FSE = „fast spin echo“, GRE = „gradient echo“, DESS = „double echo steady state“, } \\
\mathrm{VIBE}=\text {, volume interpolated breathhold excitation“ }\end{array}$} \\
\hline
\end{tabular}


- Tab. 2 Empfehlungen zur MRT der Finger bzw. des Daumens. 2-D-Schichtdicke von 2,0 oder 1,5 mm koronal und sagittal (lückenlos) sowie 2,5 oder 2,0 mm axial. 3-D-Partitionsdicke von $0,5 \mathrm{~mm}$ für die VIBE-Sequenz.

\begin{tabular}{|c|c|c|c|c|c|c|c|}
\hline Protokoll & $\begin{array}{l}\text { Indikationen an } \\
\text { Fingern und Daumen }\end{array}$ & PD FSE fs & $\begin{array}{l}\text { T1 FSE } \\
\text { nativ }\end{array}$ & $\begin{array}{l}\text { PD FSE } \\
\text { fs oder } \\
\text { T2* GRE }\end{array}$ & $\begin{array}{l}\text { T1 FSE fs } \\
\text { nach i. v. } \\
\text { Gadolinium }\end{array}$ & $\begin{array}{l}\text { T1 FSE fs } \\
\text { nach i. v. } \\
\text { Gadolinium }\end{array}$ & optional \\
\hline 7 & $\begin{array}{l}\text { Übersicht, } \\
\text { Kollateralbänder }{ }^{1}\end{array}$ & koronal & koronal & axial & koronal & sagittal & 3-D-VIBE 2 \\
\hline 8 & $\begin{array}{l}\text { Sehnen, } \\
\text { palmare Platte }{ }^{1}\end{array}$ & sagittal & sagittal & axial & sagittal & koronal & 3-D-VIBE 3 \\
\hline \multicolumn{8}{|c|}{$\begin{array}{l}{ }^{1} \text { Akquisition mit axialen Schichten beginnen, auf axialen Schichten die koronalen und sagittalen Ebenen planen. Vorsicht beim Daumen, der } 45^{\circ}-60^{\circ} \\
\text { zu den übrigen Fingern anguliert ist. }\end{array}$} \\
\hline \multicolumn{8}{|c|}{${ }^{2}$ zur Diagnostik der Kollateralbänder hilfreich; Akquisition koronal, MPR schräg-sagittal parallel zu den Kollateralbändern } \\
\hline \multicolumn{8}{|c|}{${ }^{3}$ zur Diagnostik der Extensorensehnen hilfreich; Akquisition koronal, MPR sagittal } \\
\hline \multicolumn{8}{|c|}{ PD = Protonendichte, fs = „fat saturated“, FSE = „fast spin echo“, GRE = „gradient echo“, VIBE = „,volume interpolated breathhold excitation“ } \\
\hline
\end{tabular}

- Tab. 3 Empfehlungen zur MRT der ganzen Hand. Aufgeführt sind synovialitische bzw. arthritische Erkrankungen mit Abbildung aller Handgelenke und Durchblutungsstörungen bzw. Gefäßmalformationen.

\begin{tabular}{|c|c|c|c|c|c|c|c|}
\hline Protokoll & seltene Indikationen & PD FSE fs & $\begin{array}{l}\text { T1 FSE } \\
\text { nativ }\end{array}$ & $\begin{array}{l}\text { PD FSE } \\
\text { fs oder } \\
\text { T2* GRE }\end{array}$ & $\begin{array}{l}\text { T1 FSE fs } \\
\text { nach i.v. } \\
\text { Gadolinium }\end{array}$ & $\begin{array}{l}\text { T1 FSE fs } \\
\text { nach i.v. } \\
\text { Gadolinium }\end{array}$ & MR-Angiografie \\
\hline 9 & $\begin{array}{l}\text { ganze Hand: Arthritis, } \\
\text { Synovialitis }{ }^{1}\end{array}$ & koronal & koronal & axial & koronal & - & - \\
\hline 10 & $\begin{array}{l}\text { ganze Hand: } \\
\text { MR-Angiografie, } \\
\text { Hämangiom }{ }^{1}\end{array}$ & koronal & axial & - & axial & koronal & zeitaufgelöst ${ }^{2}$ \\
\hline \multicolumn{8}{|c|}{1 Schichtdicke 3 mm koronal lückenlos und axial mit Schichtlücke } \\
\hline \multicolumn{8}{|c|}{$\begin{array}{l}2 \text { koronale Akquisition: Partitionsdicke 1,2-1,8 mm, Phasenzeit 3-5 s, koronale MIP-Rekonstruktion meist ausreichend (TRICKS, Fa. GE Healthcare; } \\
\text { CENTRA, Fa. Philips Healthcare; TWIST, Fa. Siemens Healthineers) }\end{array}$} \\
\hline \multicolumn{8}{|c|}{$\begin{array}{l}\mathrm{PD}=\text { Protonendichte, fs = „fat saturated“, FSE = „fast spin echo“, GRE = „gradient echo“, DESS = „double echo steady state“, VIBE = „volume interpolated } \\
\text { breathhold excitation“ }\end{array}$} \\
\hline
\end{tabular}

\section{Schichtebenen}

Anhand von Lokalisatoren in 3 Raumebenen werden die endgültigen Schichtebenen geplant. Für eine reproduzierbare, anatomische Ausrichtung müssen die Schichten in einer dreistufigen Korrektur in den orthogonalen Ebenen senkrecht aufeinander ausgerichtet werden [5]. Es schließt sich die Neupositionierung des Shim-Volumens an. Die 3 Standardebenen, die obligat akquiriert werden sollten, sowie 3 weitere Fakultativebenen stellen folgende anatomische Strukturen dar ( $\vee$ Tab. 1, Tab. 2):

\section{Koronalebene}

Sie gibt eine Übersicht über die Gelenke der Handwurzel sowie der Finger und bildet die Anordnung des karpalen Gefüges ab. An weiteren Strukturen stellen sich in der Koronalebene der ulnokarpale Komplex (TFCC, „triangular fibrocartilage complex"), das Lig. scapholunatum, das Lig. lunotriquetrum, die Kollateralbänder an den Fingern sowie die langen Flexoren- und Extensorensehnen dar,
Letztere häufig nur segmentweise in einer Schichtabfolge.

\section{Axialebene}

Hiermit wird das distale Radioulnargelenk optimal dargestellt, an dem Subluxationen mithilfe der sog. Mino-Linien leicht erkennbar werden. Des Weiteren können in der transversalen Ebene die Querschnitte des N. medianus und N. ulnaris, das Retinaculum flexorum sowie die Verläufe der Flexoren- und Extensorensehnen in anatomischer Relation zum Fingerskelett beurteilt werden.

\section{Sagittalebene}

Sie charakterisiert die Handwurzel (Kolinearität der mittleren Karpalsäule, Artikulation in den Radioskaphoidalund Radiolunargelenken), die Fingergelenke und den Zustand der palmaren Platten an den MCP- und PIP-Gelenken. Die Sagittalebene muss am untersuchten Fingeroder Daumenstrahl individuell ausgerichtet werden, da 
deren Extensoren- und Flexorensehnen nach proximal konvergieren [6].

\section{Schräg-sagittale Ebene}

Das Skaphoid wird hiermit in seiner Längsausdehnung erfasst [7]. Wegen der doppelobliquen Anordnung des Skaphoids muss die Ebene dreidimensional geplant werden. In gleicher Schichtung werden das Daumensattelgelenk und der Daumenstrahl sagittal mit den Flexor-pollicislongus-, Extensor-pollicis-brevis- und Extensor-pollicislongus-Sehnen abgebildet.

\section{Schräg-koronale Ebene}

Diese Ebene steht senkrecht zur schräg-sagittalen Ebene und stellt die 2. Längsebene des Skaphoids dar. Der gesamte Daumenstrahl einschließlich der STT- und Sattelgelenke werden koronal erfasst.

\section{Radiär ausgerichtete Ebenen}

Aufgrund seiner ulnaren Konvergenz auf die Fovea capitis bzw. den Processus styloideus ulnae wird der dreieckförmige Discus ulnocarpalis (TFC) in radiär rekonstruierten MPR-Schichten eines 3-D-Datensatzes mit Drehzentrum im Styloid übersichtlich dargestellt.

\section{Geometrische Akquisitionsparameter}

Das Abtastfeld (FoV) sollte zur Erzielung einer hohen InPlane-Ortsauflösung klein gehalten werden, an der Handwurzel zwischen 80 und $100 \mathrm{~mm}$ bei einer Bildmatrix von $320 \times 320$ [8]. Zur MRT eines gesamten Fingers wird ein FoV von mindestens $120 \mathrm{~mm}$ benötigt, bei fokaler Fragestellung (z. B. am ulnaren MCP-Kollateralband des Daumens) sind $80 \mathrm{~mm}$ sinnvoll.

Die Schichtdicken richten sich nach der Untersuchungsregion und der Schichtorientierung: Am Handgelenk sollten lückenlose, 2 mm dicke Koronalschichten angestrebt werden, während die Axial- und Sagittalebenen mit 2,5 oder $3 \mathrm{~mm}$ dicken Schichten abgedeckt werden, bei fokussierter Frage (z. B. am distalen Radioulnargelenk oder TFCC) auch dünner. Für die Finger- und Daumen-MRT sind koronale und sagittale Schichten von 1,5 oder $2 \mathrm{~mm}$ ohne Schichtlücke erforderlich, bei axialen Schichten ist eine Schichtdicke von $3 \mathrm{~mm}$ für ein ausreichendes Signal-Rausch-Verhältnis vonnöten.

\section{Wird Kontrastmittel benötigt?}

Die intravenöse Applikation eines gadoliniumhaltigen Kontrastmittels ist in der MRT-Diagnostik der Hand aus folgenden Gründen sinnvoll:

- Hyperämisches Synovialgewebe kann gegenüber Gelenk- oder Sehnenscheidenergüssen besser abgegrenzt werden, was für den Nachweis und die Aktivitätsbeurteilung von inflammatorischen Erkrankungen vorteilhaft ist [9]. Es sei darauf hingewiesen, dass die Parameter „Ödem“ und „Perfusion“ unterschiedliche Zustände der Pathoanatomie repräsentieren.
- Ähnlich verhält sich fibrovaskuläres und synoviales Reparationsgewebe an traumatisch geschädigten Strukturen des Gelenkbinnenraums. Es kommt am Rupturort zum lokalen Kontrastmittelenhancement und damit zur kontrastreichen Läsionsdarstellung auf T1w Bildern mit Fettsaturation. Die Evidenz dieser empirischen Beobachtung steht allerdings noch aus.

- Bei karpalen Osteonekrosen wird die Reparationszone gegenüber der direkt angrenzenden Nekrosezone in T1w Sequenzen nach Gadoliniumgabe signifikant genauer dargestellt als beim nativen Untersuchungsprotokoll [7].

- Bei Weichteiltumoren gelingt mit der Kontrastmittelapplikation die Entitätsbestimmung sicherer, insbesondere die Abgrenzung gegenüber den häufigen Ganglien.

\section{Untersuchungsprotokolle}

\section{2-D-Sequenzen}

Fast-(Turbo-)Spin-Echo-Sequenzen mit Turbofaktoren/ Echozuglängen (ETL) zwischen 3 und 7 sind Referenzstandard zur Gewebecharakterisierung ( $\triangleright$ Tab. 1, Tab. 2):

- Anstatt einer stark T2w FSE-Sequenz, wie sie in der Weichteildiagnostik Anwendung findet, wird in der Gelenkdiagnostik der Hand eine intermediär gewichtete Protonendichtesequenz mit einer Echozeit (TE) zwischen 40 und $45 \mathrm{~ms}$ sowie mit Fettsaturation (PD FSE fs) empfohlen [10]. Vorteile der Sequenzmodifikation sind die weitgehende Unabhängigkeit vom Magic-Angle-Effekt und die gute Darstellung des hyalinen Gelenkknorpels. Die Sequenz ist zudem sensitiv für Weichteil- und Knochenmarködeme.

- T1w FSE-Sequenzen (T1 FSE) gewährleisten anatomische Detailinformationen. Optimalerweise werden T1-FSE-Sequenzen an der Hand sowohl nativ als auch fettsaturiert nach intravenöser Gadoliniumgabe akquiriert.

- Für die Bildinterpretation vorteilhaft und deshalb empfohlen wird eine in der gleichen Schichtebene akquirierte Sequenztrias aus PD FSE fs + T1 FSE nativ + T1 FSE fs nach i. v. Gadolinium. Dabei ist die koronale Ebene für die karpale Übersicht, die Ligamente und den TFCC am besten geeignet, während die axiale Ebene Veränderungen am distalen Radioulnargelenk und im Karpaltunnel sowie Nervenpathologien und Tendopathien optimal darstellt, die sagittale Ebene dagegen Veränderungen am Lunatum und Gefügestörungen an der mittleren Karpalsäule.

- T2*w Gradienten-Echo-Sequenzen (T2* GRE) bleiben auf die Fraktur- und Knorpeldiagnostik sowie die Darstellung der Flexorensehnen im Karpaltunnel beschränkt. 


\section{3-D-Sequenzen}

3-D-Sequenzen mit Partitionsdicken im SubmillimeterBereich können aufgrund ihrer geringeren Kontraste die klassischen 2-D-FSE-Sequenzen an der Hand nicht ersetzen. Werden sie zusätzlich eingesetzt, sind sie hilfreich, um dreidimensional komplex verlaufende Strukturen darzustellen, z.B. die Extensor-pollicis-longus-Sehne (EPL) oder den nach ulnar konvergierenden TFCC [1]. Unterschieden werden 3-D-GRE- von 3-D-FSE-Sequenzen und Hybrid- sowie Multiechotechniken in T1-, T2-, T2* - oder gemischter Gewichtung [11]:

- T1w 3-D-GRE-Sequenzen (Typ VIBE) nach i. v. Gadoliniumgabe sind bei Läsionen der Sehnen und Ligamente wertvoll, wenn es am entzündlichen, degenerativen oder traumatischen Läsionsort zur synovialen oder fibrovaskulären Hyperämie gekommen ist ( Tab. 1). Wichtiger Einsatz sind Rupturen am Extensorenapparat, da dessen grazile Strukturen (Mittel- und Seitenzügel, Extensorenhaube) selbst mit dünnschichtigen 2-D-FSE-Sequenzen kaum darstellbar sind ( $\triangleright$ Tab. 2).

- T1w 3-D-GRE-Sequenzen weisen am TFCC ein schlechtes Signal-Rausch-Verhältnis auf [12]. Dagegen stellt sich der Discus ulnocarpalis hypointens und kontrastreich in einer T2*-betonten Hybridsequenz (Typ DESS) oder Multiechosequenz (Typ MEDIC = „multi-echo data image combination“) dar, wobei potenzielle Kontrastmittelanreicherungen in dessen Peripherie diesen Sequenzen entgehen $(\triangleright$ Tab. 1$)$.

\section{MRT-Zusatzuntersuchungen}

\section{Direkte MR-Arthrografie}

Schon vor Jahren wurde die Überlegenheit der direkten MR-Arthrografie gegenüber der nicht kontrastverstärkten MRT in der Diagnostik der intrinsischen Bänder (Lig. scapholunatum und Lig. lunotriquetrum) und des TFCC belegt [13]. Das semiinvasive Verfahren setzt eine fokussierte, klar formulierte Fragestellung vonseiten des Klinikers voraus. Für eine umfassende Abklärung wird die Drei-Kompartiment-Technik mit Füllung der radio- und mediokarpalen Gelenkräume sowie des distalen Radioulnargelenks empfohlen. Das injizierte Kontrastmittelvolumen führt zur Distanzierung sowie Kontrastverbesserung der intraartikulären Strukturen und damit zum verbesserten Nachweis von Partial- und Komplettrupturen, insbesondere wenn keine statische Instabilität vorliegt. Eine zusätzlich akquirierte 3-D-Sequenz vom Typ VIBE ist sinnvoll aufgrund der Möglichkeit, sekundäre MPR mit Ausrichtung entlang einer strukturellen Läsion zu erstellen ( $\triangleright$ Tab. 1). Die direkte CT-Arthrografie weist bei den genannten Indikationen identische Trefferquoten auf [14].

\section{MR-Angiografie}

In zeitaufgelöster Akquisitionstechnik (Typ TRICKS, CENTRA oder TWIST) können die Arterien des distalen Unterarmabschnitts, der Handwurzel und Mittelhand sowie potenziell auch der Finger abgebildet werden [15]. Voraussetzungen umfassen die Verwendung einer Mehrkanalspule, leistungsstarke Gradienten sowie eine Phasenzeit von ca. $3 \mathrm{~s}$, die durch relativ dicke Partitionsschichten von 1,2 bis 1,8 mm umsetzbar ist. Die MR-Angiografie ist gerechtfertigt zur ersten Orientierung bzw. Ausschlussdiagnostik beim Verdacht auf eine Durchblutungsstörung, kann die Katheterangiografie bei akraler Ischämie jedoch nicht ersetzen.

\section{Schlussfolgerung}

Grundlagen einer optimierten MRT-Untersuchungstechnik und damit einer korrekten Diagnosefindung sind umfassende klinische Informationen mit fokussierter Frage, die Verwendung einer dezidierten Mehrkanal-Handspule, die anatomisch basierte Wahl der Schichtebenen, hochaufgelöste Akquisitionsparameter (kleines FoV, Dünnschichten) und die Applikation von intravenösem oder intraartikulärem Kontrastmittel.

\section{Interessenkonflikt}

Der Autor gibt an, dass keine Interessenkonflikte vorliegen.

Korrespondenzadresse

Prof. Dr. med. Rainer Schmitt

Rankestraße 51 b

85051 Ingolstadt

radiodiagnostics@outlook.com

Literatur

[1] Chhabra A, Soldatos T, Thawait GK et al. Current Perspectives on the Advantages of 3-T MR Imaging of the Wrist. RadioGraphics 2012; 32: 879-896

[2] Kocharian A, Adkins MC, Amrami KK et al. Wrist: improved MR imaging with optimized transmit-receive coil design. Radiology 2002; 223: 870-876

[3] Partik B, Rand T, Pretterklieber ML et al. Patterns of gadopentetate-enhanced MR imaging of radiocarpal joints of healthy subjects. AJR Am J Roentgenol 2002; 179: 193-197

[4] Zanetti M, Hodler J, Gilula LA. Assessment of dorsal or ventral intercalated segmental instability configurations of the wrist: Reliability of sagittal MR images. Radiology 1998; 206: 339345

[5] Schmitt S. Magnetresonanztomografie. In: Schmitt R, Lanz U, Hrsg. Bildgebende Diagnostik der Hand. Stuttgart: Thieme; 2015: 89-105

[6] De Maeseneer M, Van Roy P, Jacobson JA et al. Normal MR imaging findings of the mid-hand and fingers with anatomic correlation. Eur J Radiology 2005; 56: 278-285 
[7] Schmitt R, Christopoulos G, Wagner M et al. Avascular necrosis (AVN) of the proximal fragment in scaphoid nonunion: Is intravenous contrast agent necessary in MRI? Eur J Radiol 2011; 77: 222-227

[8] Saupe N, Pfirrmann CWA, Schmid MR et al. MR imaging of cartilage in cadaveric wrists: Comparison between imaging at 1.5 and 3.0 T and gross pathologic inspection. Radiology 2007; 243: $180-187$

[9] Tehranzadeh J, Ashikyan O, Dascalos J. Advanced imaging of early rheumatoid arthritis. Radiol Clin North Am 2004; 42: 89-107

[10] Stäbler A, Spieker A, Bonel H et al. Magnetresonanztomographie des Handgelenks - Vergleich hochauflösender Pulssequenzen und unterschiedlicher Fettsignalunterdrückungen an Leichenpräparaten. Fortschr Röntgenstr 2000; 172: 168174

[11] Nitz WR. MR imaging: Acronyms and clinical applications. Eur Radiol 1999; 9: 979-997

[12] Rehnitz C, Klaan B, von Stillfried F et al. Comparison of Modern 3D and 2D MR Imaging Sequences of the Wrist at 3 Tesla. Fortschr Röntgenstr 2016; 188: 753-762
[13] Cerezal L, Abascal F, Garcia-Valtuille R et al. Wrist MR arthrography: how, why, when. Radiol Clin North Am 2005; 43: 709-731

[14] Schmid MR, Schertler T, Pfirrmann CW et al. Interosseous ligament tears of the wrist: comparison of multi-detector row CT arthrography and MR imaging. Radiology 2005; 237: 10081013

[15] Brauck K, Maderwald S, Vogt FM et al. Time-resolved contrastenhanced magnetic resonance angiography of the hand with parallel imaging and view sharing: initial experience. Eur Radiol 2007; 17: 183-192

Bibliografie

DOI https://doi.org/10.1055/a-0795-1849

Radiologie up2date 2019; 19: 9-14

(c) Georg Thieme Verlag KG Stuttgart · New York ISSN 1616-0681 